\title{
Morphomolecular pathology: setting the framework for a new generation of pathologists
}

\author{
J Louise Jones ${ }^{\star}, 1$ Karin A Oien ${ }^{2}$, Jessica L Lee ${ }^{3}$ and Manuel Salto-Tellez ${ }^{\star, 4}$ \\ ${ }^{1}$ Centre for Tumour Biology, Barts Cancer Institute, Barts and the London School of Medicine and Dentistry, London EC1M \\ 6BQ, UK; ${ }^{2}$ Institute of Cancer Sciences-Pathology, College of Medical, Veterinary and Life Sciences, University of \\ Glasgow, Glasgow G61 1QH, UK; ${ }^{3}$ National Cancer Research Institute, London EC1V 4AD, UK and ${ }^{4}$ Northern Ireland \\ Molecular Pathology Laboratory, Centre for Cancer Research and Cell Biology, Queen's University Belfast, Belfast BT7 1NN, \\ Northern Ireland
}

Molecular diagnostics and precision medicine are now a reality. With the roll-out of the 100000 Genomes Project, one of the most ambitious initiatives of its kind worldwide (Siva, 2015), pathways are being established for the adoption of genomic medicine across the National Health Service of the UK, the largest existing operation providing universal healthcare for its citizens. This requires a transformation in the way in which the NHS handles patient samples and integrates complimentary analyses on tissues to direct patient management. New approaches in molecular pathology are essential to address the challenges of this new era of genomic medicine. Indeed, how pathologists are able to transit from an approach to tissue diagnostics driven by morphology alone towards a true morphomolecular ethos (Salto-Tellez, 2007) is arguably the most important test for tissue and cellular pathologists (histopathologists or surgical pathologists and cytopathologists) in the last 60 years.

This is far from the first transformation that pathology has faced since its inception. Historically (Tweel and Taylor, 2010), pathology has evolved from a discipline based on the understanding of gross and microscopic physiopathology to an established medical diagnostic specialty. However, over the last 6 decades since the discovery of the DNA helix, the challenge to use our increased understanding of the molecular basis of disease to broaden our diagnostic armamentarium has not been embraced as promptly as it could have been (Chan and Salto-Tellez, 2012). This is in contrast with other disciplines within laboratory medicine: for example, molecular haematology is embedded within clinical haematology services, modern virology is almost exclusively molecular, and contemporary genetics has almost fully completed the transition to molecular testing. This is surprising when one takes into account the many tests already underway in tissues and cells. For example, lymphomas (Siok-Bian et al, 2008), sarcomas (Siok-Bian et al, 2008), and tumours from the central nervous system (Rushing and Wesseling, 2015) need systematic molecular testing according to the international guidelines; moreover, molecular testing of colorectal, lung, breast, and some skin cancers are the epitome of what we now call precision or stratified medicine, or therapeutic pathology (Salto-Tellez, 2013). Most of these tests are equally applicable to histology or cytology samples (Salto-Tellez, 2015). In addition, many of the emerging challenges in modern medicine need to be addressed by the interrogation of tissues and cells using a combination of morphological and molecular techniques such as next-generation sequencing in routine diagnostics (Salto-Tellez and Castro, 2014), or the delivery of cancer immunotherapy supported by robust immune biomarkers (Balar and Weber, 2017). In this evolution, pathologists need to accept that techniques that are part of our traditional armamentarium develop a new meaning, and new rules. This has already been exemplified by so-called therapeutic immunohistochemistry (Mccourt et al, 2012) such as ER, PR, c-erbB2/Her2, $\mathrm{ALK}$, and PD-L1, all of which require more stringent handling of tissues and quantitative interpretation.

Beyond the diagnostic setting, there has been renewed interest in tissue pathology in relation to what it can offer in large research environments. There is a clear realisation that effective and robust translational medicine requires large, high-quality, well-annotated tissue samples and expert pathology input. Reports indicate that pathology review as part of clinical trials can lead to a change in diagnosis sufficient to change management in up to $10 \%$ of cases (Nguyen et al, 2004), underscoring the importance of accurate

*Correspondence: Professor JL Jones; E-mail: I.j.jones@qmul.ac.uk or Professor M Salto-Tellez; E-mail: m.salto-tellez@qub.ac.uk 
histological assessment. However, beyond this traditional pathology role, professional biobanking (Lewis et al, 2016), digital pathology (Hamilton et al, 2014), pathology bioinformatics and information management (Mcart et al, 2015), and molecular pathological epidemiology (Hamada et al, 2016) are further areas where pathology input can have an impact on translational studies.

In spite of this recognition, one of the most puzzling paradoxes in pathology is how little training programmes have evolved in parallel with these needs, and failed to embrace this new paradigm of pathology: in the future morphological pathology alone is still essential but is certainly not sufficient. This established discipline can be only enhanced by the new parallel, morphomolecular pathology.

Avoiding training ( $r$ )evolution will hinder and fragment the progress of molecular diagnostics, providing a less integrated approach to molecular tissue pathology, which may not deliver the full promise of molecular medicine to our patients.

Hereby, we strongly encourage national training bodies to rise to the challenge that the 100000 Genomes Project, and many other initiatives, are now posing: the systematic and profound review of the Pathology training curriculum to adapt the new generation of pathologists to a new era. The challenge is not small. Training programmes would need to maintain their current training for primary morphologists, as this is a demand that will never end, and, in parallel, begin to deliver training programmes that will allow a cadre of trainees to pursue a true morphomolecular career. This may require a commitment to subspecialisation earlier on in training and may take the form of parallel, modular training programmes delivered by a group of specialist centres, which would provide exposure to a diversity of training. It may also require an almost one-to-one training plan and evaluation scheme. This complexity in training will be necessary to prepare our pathologists for the increased diagnostic complexity of modern medicine.

It is our view that maintaining the status quo will be detrimental for both the next generation of pathologists, for the specialty as a whole, and for the future of molecular diagnostics and precision medicine. Whether or not we embrace this radical change, the future of pathology is our responsibility. Our generation needs to carefully consider the challenge of adapting to this genomic era, and, if we do not act soon, we may be the last generation of pathologists able to do so. Embracing this challenge will prove that, once again, pathology has managed to adapt successfully to yet another transformative challenge.

\section{ACKNOWLEDGEMENTS}

This editorial is part of the work of CM-Path, a National Cancer Research Institute initiative. CM-Path aims to achieve the change needed to support academic cellular molecular pathology in the UK and make the resulting benefits available to the wider research community (https://cmpath.ncri.org.uk/).

\section{CONFLICT OF INTEREST}

The authors declare no conflict of interest.

\section{REFERENCES}

Balar AV, Weber JS (2017) PD-1 and PD-L1 antibodies in cancer: current status and future directions. Cancer Immunol Immunother 66: 551-564. Chan JY, Salto-Tellez M (2012) Opinion. Adv Anat Pathol 19: 425-426.

Hamada T, Keum N, Nishihara R, Ogino S (2016) Molecular pathological epidemiology: new developing frontiers of big data science to study etiologies and pathogenesis. J Gastroenterol 52: 265-275.

Hamilton PW, Bankhead P, Wang Y, Hutchinson R, Kieran D, Mcart DG, James J, Salto-Tellez M (2014) Digital pathology and image analysis in tissue biomarker research. Methods 70: 59-73.

Lewis C, Mcquaid S, Hamilton PW, Salto-Tellez M, Mcart D, James JA (2016) Building a 'Repository of Science': the importance of integrating biobanks within molecular pathology programmes. Eur J Cancer 67: 191-199.

Mcart DG, Blayney JK, Boyle DP, Irwin GW, Moran M, Hutchinson RA, Bankhead P, Kieran D, Wang Y, Dunne PD, Kennedy RD, Mullan PB, Harkin DP, Catherwood MA, James JA, Salto-Tellez M, Hamilton PW (2015) PICan: an integromics framework for dynamic cancer biomarker discovery. Mol Oncol 9: 1234-1240.

Mccourt CM, Boyle D, James J, Salto-Tellez M (2012) Immunohistochemistry in the era of personalised medicine. J Clin Pathol 66: 58-61.

Nguyen PL, Schultz D, Renshaw AA, Vollmer RT, Welch WR, Cote K, D'Amico AV (2004) The impact of pathology review on treatment recommendations for patients with adenocarcinoma of the prostate. Urol Oncol 22: 295-299.

Rushing EJ, Wesseling P (2015) Towards an integrated morphological and molecular WHO diagnosis of central nervous system tumors. Curr Opin Neurol 28: 628-632.

Salto-Tellez M (2007) A case for integrated morphomolecular diagnostic pathologists. Clin Chem 53: 1188-1190.

Salto-Tellez M (2013) Overview of molecular tests and personalized cancer medicine. In: Principles of Molecular Diagnostics and Personalized Cancer Medicine. Tan D, Lynch HT (eds). Wolters Kluwer Health, Lippincott, Williams and Wilkins: Philadelphia, PA, USA, pp 196-205.

Salto-Tellez M (2015) Diagnostic molecular cytopathology-a further decade of progress. Cytopathology 26: 269-270.

Salto-Tellez M, Castro DGD (2014) Next-generation sequencing: a change of paradigm in molecular diagnostic validation. J Pathol 234: 5-10.

Siok-Bian N, Lee V, Das K, Salto-Tellez M (2008) The relevance of molecular diagnostics in the practice of surgical pathology. Exp Opin Med Diagn 2: 1401-1414.

Siva N (2015) UK gears up to decode 100000 genomes from NHS patients. Lancet 385: 103-104.

Tweel JGVD, Taylor CR (2010) A brief history of pathology. Virchows Arch 457: 3-10.

(c) (i) (2) (2) This work is licensed under the Creative Commons cc. ${ }_{\mathrm{BY}} \mathrm{NC}$ SA Attribution-Non-Commercial-Share Alike 4.0 International License. To view a copy of this license, visit http:// creativecommons.org/licenses/by-nc-sa/4.0/

(C) The Author(s) named above 2017 